\title{
Reversed palmaris longus muscle: Anatomical variant - case report and literature review
}

\author{
Amera Murabit MD FRCSC, Maria Gnarra MD, Adel Mohamed MD
}

\begin{abstract}
A Murabit, M Gnarra, A Mohamed. Reversed palmaris longus muscle: Anatomical variant - case report and literature review. Can J Plast Surg 2013;21(1):55-56.
\end{abstract}

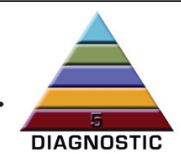

The palmaris longus, a slender fusiform muscle, is especially prone to exhibiting anatomical variance relative to other muscles in the upper extremity. The most frequent anatomical variation is the completely absent palmaris longus, followed by the reversed, duplicated, bifid or hypertrophied palmaris longus muscles. The reversed palmaris longus muscle represents a structure that is tendinous proximally and muscular distally (opposite of the normal palmaris longus). The present report describes a case of reversed palmaris longus muscle, followed by a literature review to illustrate the wide spectrum of anatomical variations in the palmaris longus muscle and their clinical and surgical relevance.

Key Words: Anatomical variations; Palmaris longus

\section{L'inversion du grand palmaire, une variante anatomique : rapport de cas et analyse bibliographique}

Le grand palmaire, un muscle fusiforme mince, est particulièrement enclin aux variations anatomiques par rapport aux autres muscles des membres supérieurs. La variation anatomique la plus fréquente est l'absence de grand palmaire, suivie de son inversion, de son dédoublement, de sa bifidité et de son hypertrophie. L'inversion du grand palmaire est une structure tendineuse sur le plan proximal et musculaire sur le plan distal (à l'opposé du grand palmaire normal). Le présent rapport décrit un cas d'inversion du grand palmaire, suivi d'une analyse bibliographique pour démontrer le large spectre de variations anatomiques du grand palmaire et leur pertinence clinique et chirurgicale.

\section{CASE PRESENTATION}

$\mathrm{R}$ outine dissection of the left upper extremity of an 82-year-old Caucasian female cadaver, performed in the Department of Anatomy at the University of Saskatchewan (Saskatoon, Saskatchewan), revealed a rare variation in the anatomy of the palmaris longus (PL) muscle.

On dissection and examination, it was discovered that the PL of the left upper extremity of this cadaver was 'reversed' - with the proximal portion being tendinous and the distal portion being muscular.

Examination of the upper extremity showed no signs of atrophy or hypertrophy, nor median or ulnar nerve entrapment in the left upper extremity. The cadaver's medical history, occupation and handedness were unknown. No anatomical variations were noted in the right upper extremity of this cadaver.

In the present case, the PL originated at the common flexor origin from the medial epicondyle. However, the origin was tendinous - a long thin tendon, instead of the normally observed PL muscle fibres.

Two-thirds down the forearm, the PL structure became more muscular in nature, with a muscle belly that spanned the distal one-third of the forearm. At the distal insertion of the PL, at the level of the wrist, the muscle fibres became tendinomembranous and continued into the palmar aponeurosis where they ultimately terminated (Figure 1).

\section{DISCUSSION}

The standard anatomical origin of the PL is the common flexor at the medial epicondyle, shared by other superficial flexors such as the flexor digitorum superficialis - located beneath the PL - and the flexor carpi radialis and flexor carpi ulnaris - between which the PL is located. The standard anatomical insertion is the palmar aponeurosis, volar to the flexor retinaculum. Although the palmar aponeurosis joins the PL tendon, the two are distinct entities, as shown by their different origin and development (1). The vascularity is most commonly supplied by branches of the ulnar artery, followed by branches of the brachial artery. Branches of the median nerve supply innervation (2).

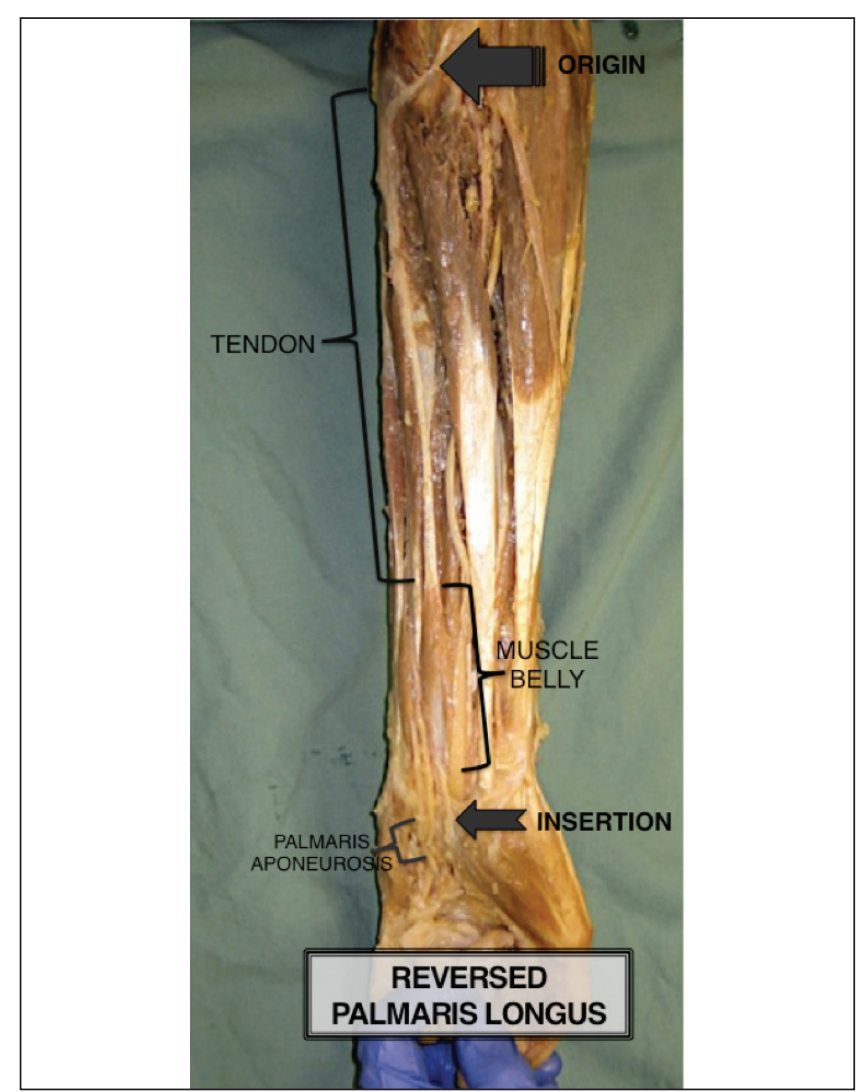

Figure 1) Anomalous reversed palmaris longus muscle found during cadaveric dissection, illustrating the palmaris longus origin, palmaris longus insertion and palmaris aponeurosis, as well as the palmaris tendinous portion (proximal) and the palmaris muscle belly (distal)

Department of Anatomy and Cell Biology, College of Medicine, University of Saskatchewan, Saskatoon, Saskatchewan

Correspondence and reprints: Dr Adel Mohamed, Department of Anatomy and Cell Biology, University of Saskatchewan, 3D01.16 Health

Science Building, 107 Wiggins Road, Saskatoon, Saskatchewan S7N 5E5. Telephone 306-966-4085, fax 306-966-4298,

e-mail adel.mohamed@usask.ca 
Several authors have described the PL muscle as a mere phylogenetically degenerate metacarpophalangeal flexor because of its clinical insignificance and its numerous anatomical variations (3-5). However, the less common variants can lead to pathological conditions due to the narrow topographical relationship between the PL and the median nerve, causing median nerve compression (6).

Agenesis of PL has been described as the most common anatomical variation, observed in approximately $2 \%$ to $25 \%$ of the population, with a higher prevalence in Caucasians (7-9).

All other anatomical variants combined account for those found in approximately $9 \%$ of the population (8). These include duplicated or bifid PL (depending on whether they share a common origin and muscle belly); triple-headed PL muscles; accessory PL; palmaris profundus (distal tendon passes deep to the flexor retinaculum); and reversed PL (9).

In the present case, the PL had a long tendinous origin proximally and a muscular portion distally. Since the initial description of a reversed PL in 1916 (10), several cases have been reported in the literature together with the clinical implications (11-14).

The reversed muscle belly can divide into separate muscular slips, which can insert into the flexor retinaculum, or onto the flexor carpi radialis tendon and volar carpal ligament (15). Thus, patients with a reversed PL may present with development of edema and inflammation accompanied by a bluish discolouration leading to numbness or pain in the volar aspect of the distal forearm. This results in a reduction of hand function usually exacerbated by wrist flexion (9).

\section{REFERENCES}

1. Caughell KA, McFarlane RM, McGrouther DA, Martin AH. Developmental anatomy of the palmar aponeurosis and its relationship to the palmaris longus tendon. J Hand Surg (Am) 1988;13:485-93.

2. Wafae N, Itezerote AM, Laurini Neto H. Arterial branches to the palmaris longus muscle. Morphologie 1997;81:25-8.

3. McMinnRMH, ed. Last's Anatomy: Regional and Applied, 9th edn. London: Churchill Livingstone; 1994:89.

4. Williams PL, Bannister LH, Dyson M, et al, eds. The Muscular System. In: Gray's Anatomy: The Anatomical Basis of Medicine and Surgery, 38th edn. London: Churchill Livingstone; 1995:846.

5. Zeybek A, Gurunluoglu R, Cavdar S, Bayramicli M. A clinical reminder: A palmaris longus muscle variation. Ann Plast Surg 1998;41:224-5.

6. Blackhouse KM, Churchill-Davidson D. Anomalous palmaris longus muscle producing carpal tunnel-like compression. Hand 1975;7:22-4.

7. Wheeless CR. Palmaris Longus. 1996 [updated 2012 Apr 10;]. $<$ www.wheelessonline.com/ortho/palmaris_longus> (Accessed December 5, 2012).

8. Reimann AF, Daseler EH, Anson BJ, Beaton LE. The palmaris longus muscle and tendon: A study of 1600 extremeties. Anat Rec 1944;89:495-505.

9. Park MJ, Namdari S, Yao J. Anatomic variations of the palmaris longus muscle. Am J Orthop (Belle Mead NJ) 2010;39:89-94.

10. Morrison JT. A palmaris longus muscle with a reversed belly forming an accessory flexor muscle of the little finger. J Anat Physiol 1916;50:324-6.
Additionally, repetitive motions can cause hypertrophy of the muscle belly of a reversed PL and, due to the unyielding nature of the antebrachial fascia, may lead to an effort-related compartment syndrome (16).

Compression of the ulnar nerve (Guyon's syndrome) associated with the reversed PL variation, although less common, has also been reported (17).

Understanding the anatomical variations of the PL is important because it often plays a crucial role during reconstructive surgeries due to its suitable length and diameter, ease of harvest and lack of donor site morbidity (18).

It is essential to clinically examine reconstructive patients for potential PL anatomical variations to avoid inappropriate surgical procedures and prevent disappointing surgical results.

\section{KEY POINTS}

- The PL is one of the anatomical structures most prone to exhibiting variance in the human body.

- Understanding the anatomical variations of the PL is essential due to its crucial role during surgery as a landmark guide and as a reconstructive tool.

DISCLOSURES: The authors have no financial disclosures or conflicts of interest to declare.

11. Bencteux P, Simonet J, el Ayoubi L, et al. Symptomatic palmaris longus muscle variation with MRI and surgical correlation: Report of a single case. Surg Radiol Anat 2001;23:273-5.

12. Depuydt KH, Schuurman AH, Kon M. Reversed palmaris longus muscle causing effort-related median nerve compression. J Hand Surg Br 1998;23:117-9.

13. Schuurman AH, van Gils AP. Reversed palmaris longus muscle on MRI: Report of four cases. Eur Radiol 2000;10:1242-4.

14. Yildiz M, Sener M, Aynaci O. Three-headed reversed palmaris longus muscle: A case report and review of the literature. Surg Radiol Anat 2000;22:217-9.

15. Acikel C, Ulkur E, Karagoz H, Celikoz B. Effort-related compression of median and ulnar nerves as a result of reversed three-headed and hypertrophied palmaris longus muscle with extension of Guyon's canal. Scand J Plast Reconstr Surg Hand Surg 2007;41:45-7.

16. Martens MA, Moeyersoons JP. Acute and recurrent effort-related compartment syndrome in sports. Sports Med 1990;9:62-8.

17. Regan PJ, Roberts JO, Bailey BN. Ulnar nerve compression caused by a reversed palmaris longus muscle. J Hand Surg $\mathrm{Br}$ 1988;13:406-7.

18. Sebastin SJ, Lim AY, Bee WH, Wong TC, Methil BV. Does the absence of the palmaris longus affect grip and pinch strength? J Hand Surg Br 2005;30:406-8. 